\title{
PREVALENCE OF ACSL (RS6552828) POLYMORPHISM AMONG RUNNERS
}

\author{
Sigal Ben-Zaken ${ }^{1}$, Yoav Meckel ${ }^{1}$, Dan Nemet ${ }^{2}$, Alon Eliakim ${ }^{2}$ \\ ${ }^{1}$ The Zinman College of Physical Education and Sports Sciences at the Wingate \\ Institute, Genetics and Molecular Biology Laboratory, Israel \\ ${ }^{2}$ Meir Medical Center, Child Health and Sports Center, Pediatric Department, \\ Sackler School of Medicine, Tel-Aviv University, Israel
}

\begin{abstract}
The ACSL A/G polymorphism is associated with endurance trainability. Previous studies have demonstrated that homozygotes of the minor AA allele had a reduced maximal oxygen consumption response to training compared to the common GG allele homozygotes, and that the ACSL A/G single nucleotide polymorphism explained $6.1 \%$ of the variance in the $\mathrm{VO}_{2}$ max response to endurance training. The contribution of ACSL single nucleotide polymorphism to endurance trainability was shown in nonathletes, however, its potential role in professional athletes is not clear. Moreover, the genetic basis to anaerobic trainability is even less studied. Therefore, the aim of the present study was to examine the prevalence of ACSL single nucleotide polymorphism among professional Israeli long distance runners $(n=59)$, middle distance runners $(n=31)$, sprinters and jumpers $(n=48)$ and non-athletic controls $(n=60)$. The main finding of the present study was that the ACSL1 AA genotype, previously shown to be associated with reduced endurance trainability, was not higher among sprinters and jumpers (15\%) compared to middle- (16\%) and long-distance runners (15\%). This suggests that in contrast to previous studies indicating that the ACSL1 single nucleotide polymorphism may influence endurance trainability among non-athletic individuals, the role of this polymorphism among professional athletes is still not clear.
\end{abstract}

Keywords: sprinters, long distance runners, genetic polymorphism, ACSL 


\section{INTRODUCTION}

Long-chain-fatty-acid - CoA ligase 1 is an enzyme that in humans is encoded by the ACSL1 gene $[12,13]$. ACSL1 exhibits an important potential relevance to the adaptation to regular exercise. ACSL1 contributes most of the acyl-CoA synthetase activity in adipose tissue and is highly expressed also in skeletal muscles and in the heart. ACSL1 encodes for an enzyme that is involved in the partitioning of fatty acids and the control of lipid influx but, even more importantly, the control of carbohydrate efflux [8]. It plays a key role in normal insulin metabolism and fatty acid-induced insulin resistance. A SNP in ACSL1 has been associated with fasting glucose, insulin resistance, and the presence of metabolic syndrome, particularly in the presence of a high-fat diet [10]. Besides its possible relevance to habitual physical activity and exercise, studies have shown that homozygotes of the rs6552828 minor allele (A/A) had $125 \mathrm{ml} / \mathrm{min}(28 \%)$ and $63 \mathrm{ml} / \mathrm{min}$ (17\%) lower $\mathrm{VO}_{2}$ max responses to endurance exercise training than the common allele homozygotes (G/G) and heterozygotes (A/G), respectively. In a single-SNP analyses, rs6552828 explained $6.1 \%$ of the variance in the $\mathrm{VO}_{2}$ max response to training [3]. Therefore, the potential contributing role of the ACSL1 gene in aerobic metabolism at the adypocite, cardiomyocite, liver and skeletal muscle fiber level $[4,5-7,9,11]$ highlights its potential to explain individual variability in endurance performance, as well as in some health-related phenotypes.

While the contribution of ACSL1 single nucleotide polymorphism to endurance trainability was shown in non-athletes, its potential role in professional athletes is less studied. Moreover, the genetic basis to anaerobic trainability is even less studied. Therefore, the aim of the present study was to examine the prevalence of ACSL1 SNP among professional Israeli long distance runners, middle distance runners, sprinters and jumpers. We hypothesized that the ACSL1 AA genotype associated with reduced endurance trainability would be higher among sprinters and jumpers.

\section{MATERIALS AND METHODS}

\section{Subjects}

One hundred and thirty-eight male athletes [59 long distance runners (LDR), 31 middle distance runners (MDR), 48 sprinters and jumpers (S/J)], and 60 non-athletic controls participated in the study. All athletes competed in national and/or international level meets on a regular basis. Athletes' data are presented in Table 1. 
Table 1. Athletes' data.

\begin{tabular}{lccc}
\hline Group & $\mathbf{N}$ & Main even & Age (Mean \pm SD, Range) \\
\hline LDR & 59 & $5000 m$-marathon & $32.4 \pm 9.5(20-50)$ \\
\hline MDR & 31 & $800-1500 m$ & $32.3 \pm 15.0(17-55)$ \\
\hline S/J & 48 & $100-200 m$, jumps & $30.5 \pm 13.7(17-49)$ \\
\hline Control & 60 & $n r$ & $26.4 \pm 5.8(19-29)$ \\
\hline
\end{tabular}

LDR - long distance runners; MDR - middle distance runners; $S / J$ - sprinters and jumpers

The study was approved by the Institutional Review Board of the Hillel Yaffe Medical Center, Hadera, Israel, according to the Declaration of Helsinki. A written informed consent was obtained from all participants.

\section{Genotyping}

Genomic DNA was extracted from samples of peripheral venous blood or buccal cells according to the salting-out procedure. Genotypes were determined using the Taqman allelic discrimination assay. The Assay-by-Design service (https://www.thermofisher.com /il/en/home.html) was used to set up a Taqman allelic discrimination assay for the ACSL (rs6552828 A/G), Primers and probe sequences are given in Table 2.

Table 2. Primers and probe sequences for ACSL (rs6552828 A/G) Taqman allelic discrimination assays.

\begin{tabular}{lcll}
\hline \multicolumn{2}{c}{ Primer sequences } & \multicolumn{2}{c}{ Probe sequences } \\
\hline \multicolumn{1}{c}{ Forward } & \multicolumn{1}{c}{ Reverse } & \multicolumn{1}{c}{ Forward:VIC } & \multicolumn{1}{c}{ Reverse: FAM } \\
\hline pdUGpdUApdCpd & pdCpdUGpdUApdC & CGGCTGCATCTA & TGCATAAACTTT \\
UpdUpdUpdCpdUp & pdUpdUpdUpdCpd & GGATCTCAA & AAACCAACCAC \\
dCpdUpdCpdCAAp & UpdUpdUpdCpdCA & & CA \\
dCA & ApdCA & & \\
\hline
\end{tabular}

The Polymerase Chain Reaction (PCR) mixture included 5ng genomic DNA, $0.125 \mu \mathrm{l} \mathrm{TaqMan}$ assay (40*, ABI), $2.5 \mu \mathrm{l}$ Master mix (ABI) and $2.375 \mu \mathrm{l}$ water. PCR was performed in 96 well PCR plates in an ABI 7300 PCR system (Applied Biosystems Inc., Foster City, CA, USA) and consisted of initial denaturation for $5 \mathrm{~min}$ at $95{ }^{\circ} \mathrm{C}$, and 40 cycles with denaturation of $15 \mathrm{~s}$ at $95^{\circ} \mathrm{C}$ and annealing and extension for 60 s at $63{ }^{\circ} \mathrm{C}$. Results were analyzed by the ABI Taqman $7900 \mathrm{HT}$ using the sequence detection system 2.22 software (Applied Biosystems Inc., Foster City, CA, USA). 


\section{Statistics}

The SPSS statistical package, version 20.0, was used to perform all statistical evaluations (SPSS, Chicago, IL, USA). A $\chi 2$-test was used to confirm that the observed genotype frequencies were within the Hardy-Weinberg equilibrium, and to compare allele and genotype frequencies between athletes and controls, as well as between athletes from different competitive levels. If observed or expected values included a cell with a value of 5 , we used Fisher's exact test to compare alleles and genotype frequencies.

\section{RESULTS}

The complete data on allele and genotype frequencies are presented in Table 3. The genotype subtype did not differ by age or sex. ACSL genotypes were in agreement with the Hardy-Weinberg equilibrium for all groups ( $\mathrm{p}=0.917$ for Controls, $\mathrm{p}=0.843$ for $\mathrm{S} / \mathrm{J}, \mathrm{p}=0.856$ for MDR; $\mathrm{p}=0.930$ for LDR).

Table 3. ACSL A/G (rs6552828) Genotype and allele frequencies (\%).

\begin{tabular}{lcccc}
\hline & Control & S/J & MDR & LDR \\
\hline $\mathrm{n}$ & 60 & 48 & 31 & 59 \\
\hline $\mathrm{AA}$ & $7(12)$ & $7(15)$ & $5(16)$ & $9(15)$ \\
\hline $\mathrm{AG}$ & $24(40)$ & $23(48)$ & $11(35)$ & $30(51)$ \\
\hline $\mathrm{GG}$ & $29(48)$ & $18(38)$ & $15(48)$ & $20(34)$ \\
\hline A allele & $38(32)$ & $37(39)$ & $21(34)$ & $48(41)$ \\
\hline G allele & $82(68)$ & $59(61)$ & $41(66)$ & $70(59)$ \\
\hline
\end{tabular}

$\mathrm{S} / \mathrm{J}$ - sprinters and jumpers; MDR - middle distance runners; LDR - long distance runners

The frequency of the ACSL AA genotype carriers, which is related to reduced $\mathrm{VO}_{2}$ max response to training, was not significantly different among LDR, MDR, S/J and controls (Figure 1). 


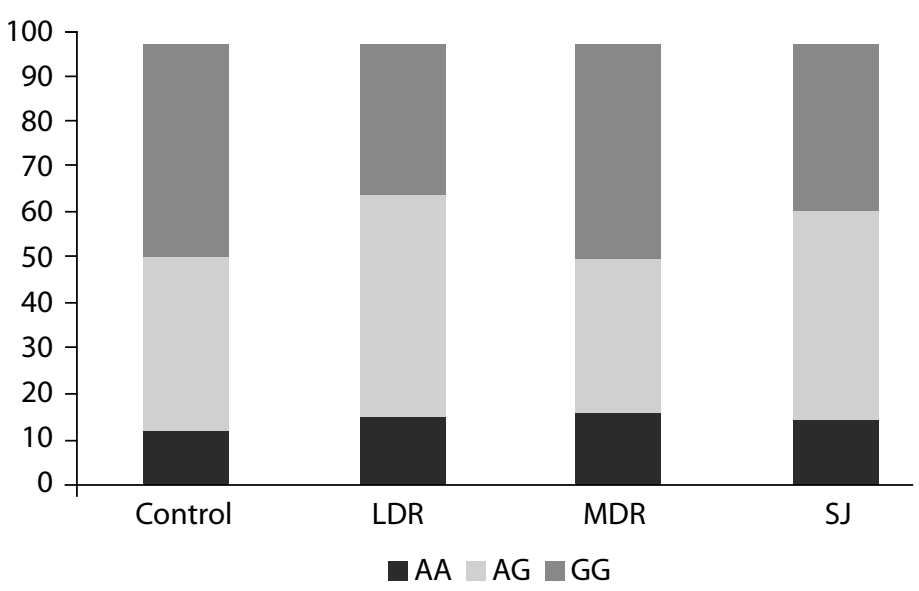

Figure 1. ACSL A/G genotype frequencies among $S / J, M D R, L D R$, and controls.

\section{DISCUSSION}

The present study examined the prevalence of ACSL1 SNP among professional Israeli long distance runners, middle distance runners, sprinters and jumpers. The main finding of the study was that in contrast to our hypothesis, the ACSL1 AA genotype, previously shown to be associated with reduced endurance trainability, was not higher among sprinters and jumpers compared to long-distance runners.

The ACSL A/G polymorphism is associated with endurance trainability. It was previously demonstrated that homozygotes of the minor AA allele had a reduced $\mathrm{VO}_{2}$ max response to training compared to the common GG allele homozygotes, and that the ACSL A/G single nucleotide polymorphism explained $6.1 \%$ of the variance in the $\mathrm{VO}_{2} \max$ response to endurance training [3]. However, while this association was found in non-athletic individuals, the linkage between the ACSL1 single nucleotide polymorphism and endurance trainability among professional athletes, and in particularly, among a homogeneous group of long-distance runners, is much less clear.

The ACSL SNP may vary among different populations of long-distance runners. Consistent with that the frequency of the AA genotype (associated with reduced endurance trainability) among native Israeli long-distance runners was higher $(24 \%)$ than the frequencies reported among Spanish endurance athletes (17.1\%) and lower compared to Chinese $(31.3 \%)$ endurance athletes [14]. Moreover, we recently studied the frequency of the ACSL genotype among Israeli long-distance runners of Ethiopian and nonEthiopian origin. The Ethiopian Beta Israel community consist only about 
$1.7 \%$ of the overall Israeli population, but their dominance in Israeli longdistance record table is dramatic (e.g. between $50-66 \%$ of the 30 all-time best male results in the marathon and half-marathon). We found that the frequency of the AA genotype carriers among Israeli long-distance runners of Ethiopian origin was significantly lower (8\%) compared to the nonEthiopian Origin Israeli runners (24\%) [2]. Thus, fewer long-distance runners of Ethiopian origin had a genetic predisposition for reduced response to aerobic training. Interestingly, among the non-Ethiopian origin runners the frequency of AA carriers was significantly lower among elite compared to national levels runners suggesting the potential role of the ACSL genotype for endurance excellence in this population as well.

We hypothesized that since endurance trainability is less important for short-distances athletic events, the frequency of the ACSL1 single nucleotide polymorphism would be lower among sprinters and jumpers. However, in contrast to our hypothesis no significant difference was found in the ACSL AA genotype between professional Israeli long-distance runners, middledistance runners, sprinters and jumpers. The potential contribution of many potential genes for excellence in endurance and anaerobic-power type sports were studied in recent years, and several favoring endurance and anaerobicpower single nucleotide polymorphism profiles were proposed [1]. The present study suggests that the ACSL1 single nucleotide polymorphism cannot distinguish between these two ends of the endurance to sprint

The potential use of genetic polymorphisms, and in particular the single nucleotide polymorphism (SNP) of hormone genes, as a tool to assist in predicting future athletic performance is currently an extremely challenging topic, mainly because each possible gene makes only a small contribution to the overall heritability. Previous results and the results of the present study suggest that while the ACSL1 single nucleotide polymorphism may influence endurance trainability among non-athletic individuals, its role among professional athletes is still not clear. 


\section{REFERENCES}

1. Ben-Zaken S, Meckel Y, Nemet D, Eliakim A. (2015) Genetic score of powerspeed and endurance track and field athletes. Scand J Med Sci Sports, 25: 166174.

2. Ben-Zaken S, Meckel Y, Nemet D, Kassem E, Eliakim A. (2018) Genetic basis for the dominance of Israeli long-distance runners of Ethiopian origin. J Strength Cond Res Epub.

3. Bouchard C, Sarzynski MA, Rice TK, Kraus WE, Church TS, Sung YJ, Rao DC, Rankinen T. (1985) Genomic predictors of the maximal $\mathrm{O}_{2}$ uptake response to standardized exercise training programs. J Appl Physiol, 110: 1160-1170.

4. Coleman RA, Lewin TM, Muoio DM. (2000) Physiological and nutritional regulation of enzymes of triacylglycerol synthesis. Annu Rev Nutr, 20: 77-103.

5. Ellis JM, Li LO, Wu PC, Koves TR, Ilkayeva O, Stevens RD, Watkins SM, Muoio DM, Coleman RA. (2010) Adipose acyl-CoA synthetase-1 directs fatty acids toward beta-oxidation and is required for cold thermogenesis. Cell Metab, 12: 53-64.

6. Hall AM, Smith AJ, Bernlohr DA. (2003) Characterization of the Acyl-CoA synthetase activity of purified murine fatty acid transport protein 1 . J Biol Chem, 278: 43008-43013.

7. Martin G, Schoonjans K, Lefebvre AM, Staels B, Auwerx J. (1997) Coordinate regulation of the expression of the fatty acid transport protein and acyl-CoA synthetase genes by PPARalpha and PPARgamma activators. J Biol Chem, 272: 28210-28217.

8. Mashek DG, Li LO, Coleman RA. (2007) Long-chain acyl-CoA synthetases and fatty acid channeling. Future Lipidol, 2: 465-476.

9. Mashek DG, Li LO, Coleman RA. (2006) Rat long-chain acyl-CoA synthetase mRNA, protein, and activity vary in tissue distribution and in response to diet. J Lipid Res, 47: 2004-2010.

10. Phillips CM, Goumidi L, Bertrais S, Field MR, Cupples LA, Ordovas JM, Defoort C, Lovegrove JA, Drevon CA, Gibney MJ, Blaak EE, Kiec-Wilk B, Karlstrom B, Lopez-Miranda J, McManus R, Hercberg S, Lairon D, Planells R, Roche HM. (2010) Gene-nutrient interactions with dietary fat modulate the association between genetic variation of the ACSL1 gene and metabolic syndrome. J Lipid Res, 51: 1793-1800.

11. Schoonjans K, Staels B, Auwerx J. (1996) Role of the peroxisome proliferatoractivated receptor (PPAR) in mediating the effects of fibrates and fatty acids on gene expression. J Lipid Res, 37: 907-925.

12. Stanczak H, Stanczak JJ, Singh I. (1992) Chromosomal localization of the human gene for palmitoyl-CoA ligase (FACL1). Cytogenet Cell Genet. 59: 17-19.

13. Suzuki, H. Kawarabayasi Y, Kondo J, Abe T, Nishikawa K, Kimura S, Hashimoto T, Yamamoto T. (1990) Structure and regulation of rat long-chain acyl-CoA synthetase. J Biol Chem, 265: 8681-8685. 
14. Yvert T, He ZH, Santiago C, Hu Y, Li YC, Gómez-Gallego F, Fiuza-Luces C, Verde Z, Muniesa CA, Oliván J, Santalla A, Ruiz JR, Lucia A. (2012). Acyl coenzyme a synthetase long-chain 1 (ACSL1) gene polymorphism (rs6552828) and elite endurance athletic status: a replication study. PLoS One, 7: e41268.

\section{Correspondence to:}
Alon Eliakim
Meir Medical Center
Pediatric Department
Sackler School of Medicine
Tel-Aviv University, Israel
E-mail: Eliakim.alon@clalit.org 